\title{
More Than a Mission - Modelling the Impact of a Support Solution on Submarine Availability, Cost, and Safety
}

\author{
S K Crawford, BEng (Hons) MIET, R J C MacMillan, MEng (Hons) AMIMechE \\ Babcock International Group ${ }_{\circledast}$
}

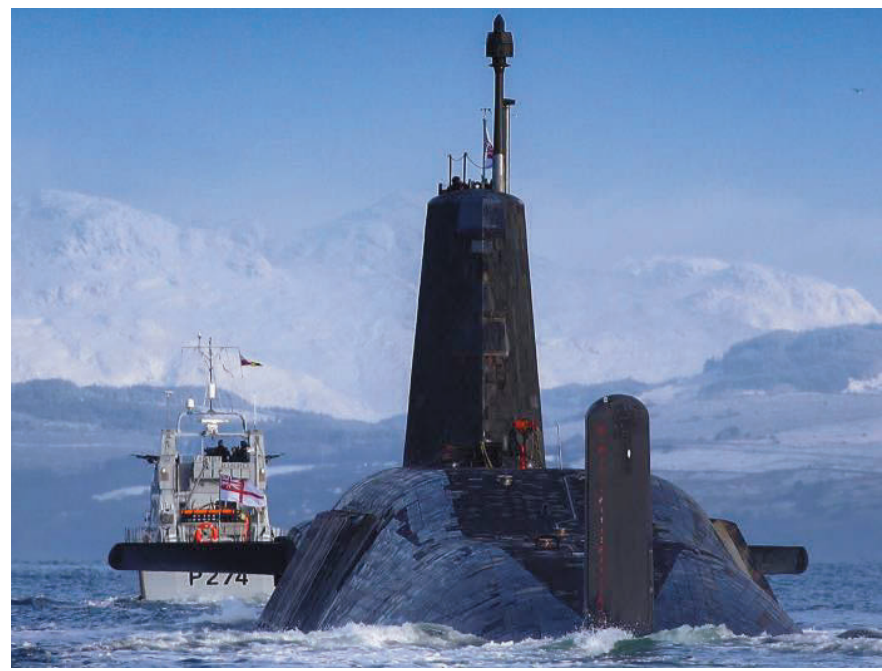

\section{Synopsis}

With the demand for increased availability and a higher level of safety, the defence industry is faced with reducing operational and maintenance budgets. Additional focus on acquisition programmes is being placed on through-life requirements, with demonstration and assurances sought early in the life cycle that these requirements can be achieved. Generic approaches to availability, cost and safety modelling are being reexamined, with a push to provide a universal view across both the submarine life-cycle and the support enterprise.

The role of a support solution is to ensure continuous understanding of the platform material state, thereby maintaining operational readiness, and to do so in a safe and cost effective manner. Despite the fact that the mission only represents a portion of the life of a platform, traditional availability studies have tended to focus on the performance in this state, thus neglecting the impact that standby and maintenance periods may have. This paper looks beyond the inherent design characteristics of a platform and towards the other enterprise factors that may affect availability, with a view to modelling and quantifying the impact these influences may have on overall platform availability.

A collaborative approach is described, with data being drawn from a number of organisations at various maturity levels and integrated into a cohesive, class level model. Targets are derived and reports produced that enable interrogation of driving factors down to a low level of granularity across multiple areas, including material failure modes, training provision, facilities, infrastructure and technical publications.

Modelling and simulation has been used in order to forecast the ability of a product to meet availability, safety and cost requirements when operated in a defined usage and upkeep cycle. These predictions, performed as early in the design phase as possible, enable the macro effects of small design changes to be assessed and feedback given into design teams. As the design phase progresses, outputs are used to optimise decisions made in the support solution design against constraints in the platform design - with the ultimate aim of maximizing capability whilst working within greater financial constraints. The development of this process and model is intended to provide increased confidence that an available, safe and affordable platform will be delivered.

Keywords: Support solution; Submarine; Availability; Safety; Cost

\footnotetext{
Author's biography

Mr Scott Crawford and Mr Robert MacMillan are presently working within the Whole Boat Support Integrator (WBSI) Engineering Management team, within the Babcock Engineering Services ${ }_{\circledast}$ business unit in Bristol. Both work on a Future Submarine programme, providing assurance to the Ministry of Defence (MOD), that the platform will be available, safe, affordable and capable. Previous experience has included a number of submarine and surface ships projects both in-service and in the design phase. Scott was seconded to work with Babcock's joint venture with the Oman Drydock Company ${ }_{\circledast}$ (ODC) in Duqm, Oman, and Rob was seconded to work with General Dynamics Electric Boat ${ }_{\circledast}($ GDEB), in Connecticut, USA.
} 


\section{Introduction}

The presence of a modern, technologically advanced and capable submarine force provides a credible deterrent to those who would seek to disrupt our prosperity and way of life. They underpin a nation's safety and security, and provide key tactical and strategic capabilities. This is especially important for the UK, as an island nation with $95 \%$ of trade requiring access to the sea, and is emphasised in the 2015 UK Strategic Defence Security Review (SDSR) (HM Government, 2015) which states that submarines "remains essential" to our security.

This necessitates a platform that is not only capable when on mission, but that each one is also consistently kept within the required time to readiness between missions; whether on standby or in a maintenance period. To meet these demands, it is far greater than just sending submarines out on patrol, and requires a full support solution to make sure each platform can complete their missions successfully. The role of a support solution is to ensure operational readiness and maintain a continuous understanding of the platform's material state. This enables effective decision making to achieve the required through-life targets for:

- $\quad$ Availability - to perform its functions

- Safety - for operators, maintainers, and civilians

- Affordability - to remain within financial constraints

Acquisition programmes are increasingly focused on assuring and demonstrating that the through-life requirements can be achieved. Modelling and simulation has been performed as early in the design phase as possible in order to forecast the ability of a product to meet these requirements when operated in a defined usage and upkeep cycle. This enables the macro effects of small design changes to be assessed and feedback given into design teams. Traditional focus is on platform mission performance, neglecting the effects that lower readiness states and the wider support enterprise may have on availability between missions. Quantifying the impact these influences may have on overall platform availability, and including them in the development of this process and model, is intended to provide increased confidence that an available, safe and affordable platform will be delivered.

The greater the level of detail built into the model, the more representative the forecast will be of platform or class availability. An Intrinsic Availability $\left(\mathrm{A}_{\mathrm{i}}\right)$ model only takes into account Corrective Maintenance $(\mathrm{CM})$ downtime from the design Availability, Reliability \& Maintainability (AR\&M) performance. An Achieved Availability $\left(\mathrm{A}_{\mathrm{A}}\right)$ model also takes into account Preventive Maintenance (PM) downtime. An Operational Availability $\left(\mathrm{A}_{\mathrm{O}}\right)$ model is as close as feasible to a measure of the "real" average availability and includes all sources of downtime from the design and the support solution.

$$
\begin{aligned}
& \text { Intrinsic Availability Achieved Availability Operational Availability } \\
& A_{i}=\frac{\text { Up Time }}{\text { Up Time }+C M} \quad A_{A}=\frac{U p \text { Time }}{\text { Up Time }+C M+P M} \quad \mathrm{~A}_{0}=\frac{\text { Up Time }}{\text { Up Time }+ \text { Total Downtime }}
\end{aligned}
$$

Figure 1 Availability Definitions

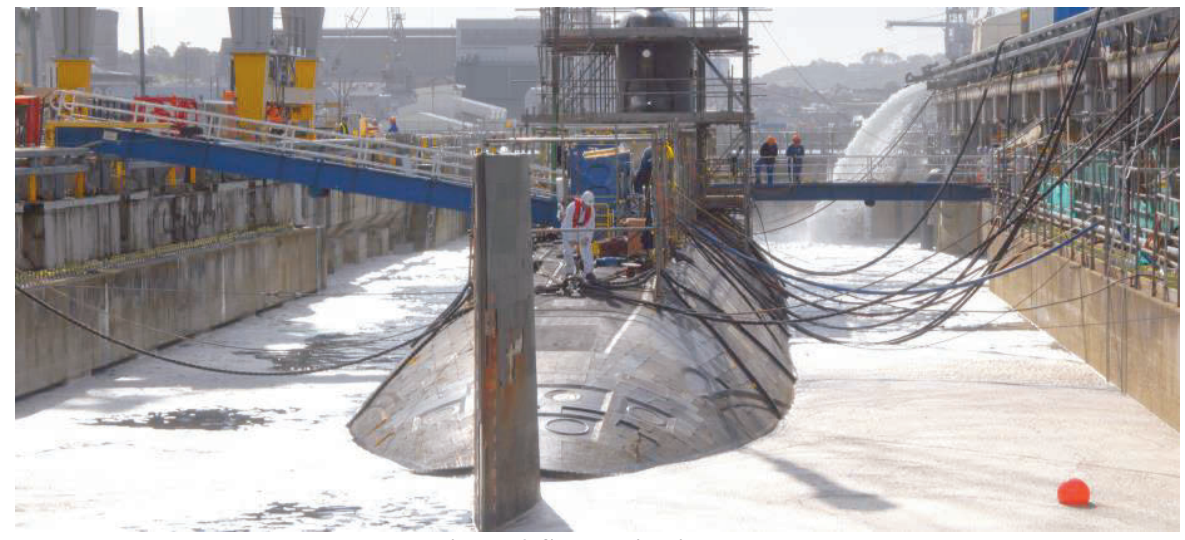

Figure 2 Submarine in dock 
It must be taken into account that, on the whole, submarines do not act in isolation in their theatre of operation; multiple submarines can be required to perform a defined mission or set of missions across an upkeep cycle, to consistently deliver key tactical and strategic capabilities. In these instances the measure of availability can be extended to forecast the ability of multiple platforms to deliver the desired capability. To replicate this in a model to determine class level availability, multiple platforms are represented performing a defined class level Maintenance Upkeep and Operating Cycle (MUOC), and the interactions between platforms, known as "class logic" has been quantified.

This paper provides an overview of the process and methodology to develop an availability model, to forecast the ability of a product to meet the through-life requirements, and how outputs are used to optimise decisions made in the platform and support solution against design constraints, with the ultimate aim of maximising capability whilst working within greater financial constraints. The support solution aspects will be expanded on in a future iteration of the paper.

\section{Modelling Objectives}

The use of modelling and simulation to digitally assess and optimise platform performance is being implemented more widely across the maritime sector; such as by Kongsberg ${ }^{\circledR}$ with K-Sim ${ }^{\circledR}$ (Kongsberg, 2017). In the case of a submarine, modelling the platform design and support solution provides assurance that throughlife targets will be met and the required operational readiness will be achieved. Modelling also enables multiple scenarios and configurations to be rapidly assessed, allowing for optimisation against constraints.

The overall aim of this model is to generate output that can be used to optimise decisions made in the platform and support solution against design constraints, maximising capability whilst working within greater financial constraints. Early design decisions determine the supportability of a platform, and given that cost of a design change rises over time (Mani, 2011), as shown in Figure 4, it is advantageous to incorporate modelling into the design process as early as possible. This is true for both new submarine design programmes, and for modifications and upgrade programmes for in-service platforms.

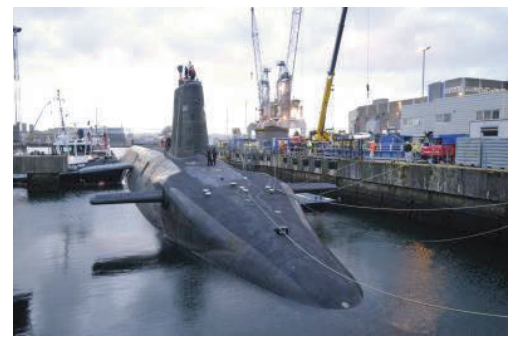

Figure 3 HMS Vengeance Preparing to Leave Devonport

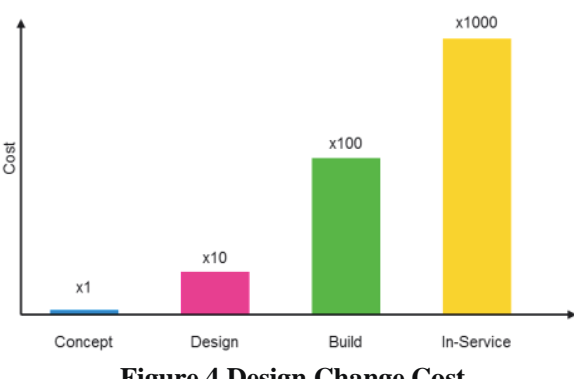

Figure 4 Design Change Cost

For a model to be effective in practice, it must balance complexity with resource restrictions, both financial and technological, to deliver the desired outputs. The business objective must be clearly defined at each level, so as the model can be tailored to maximise efficiency and usability. At class level, the model objectives are:

- For a specified platform configuration, enabling forecast of the submarine class's ability to meet the assigned through-life targets, of availability, cost and safety, to deliver the required capability throughout a specified maintenance, upkeep and operational cycle (MUOC) scenario over a defined period

- Assess the ability of the class to adhere to the specified MUOC scenario

- Identify and prioritise the greatest risk drivers, in terms of impact on through-life targets, from the platform and support solution, to provide an evidence base for key decisions to be made to maximise capability and minimise cost

For availability and safety, target compliance is assessed by forecasting the operational capability downtime the class/platform will be subjected to over a specified time period (e.g. a commission), caused by failure modes that result in the inability of a systems to perform either mission or safety critical functions. Driving systems are those with failure modes that have the largest impact. For cost, target compliance is assessed by forecasting the personnel and materials cost to maintain operational capability, for a given period. To achieve this, the model must be able to:

- Model a taskforce of platforms executing a defined MUOC scenario

- Model the AR\&M and cost characteristics of all mission critical systems within each platform and the support solution 
- Simulate the use of support resources by the taskforce, including manpower and resource requirements and constraints

- Simulate spares consumption and constraints when Corrective and Preventative Maintenance (CM and PM) are carried out at system, platform, and class level

- Model delays to the completion of PM and CM tasks caused by the design, such as work in way, and support solution, such as supply chain issues (logistics delay time) and infrastructure

The class level model is at the top of the modelling hierarchy, requiring prerequisite inputs from system and component level analysis, as illustrated in Figure 5. To generate the model inputs, the data elements that define platform and support solution AR\&M and cost characteristics a tailored programme of supportability analysis is required, which is detailed in the next section.

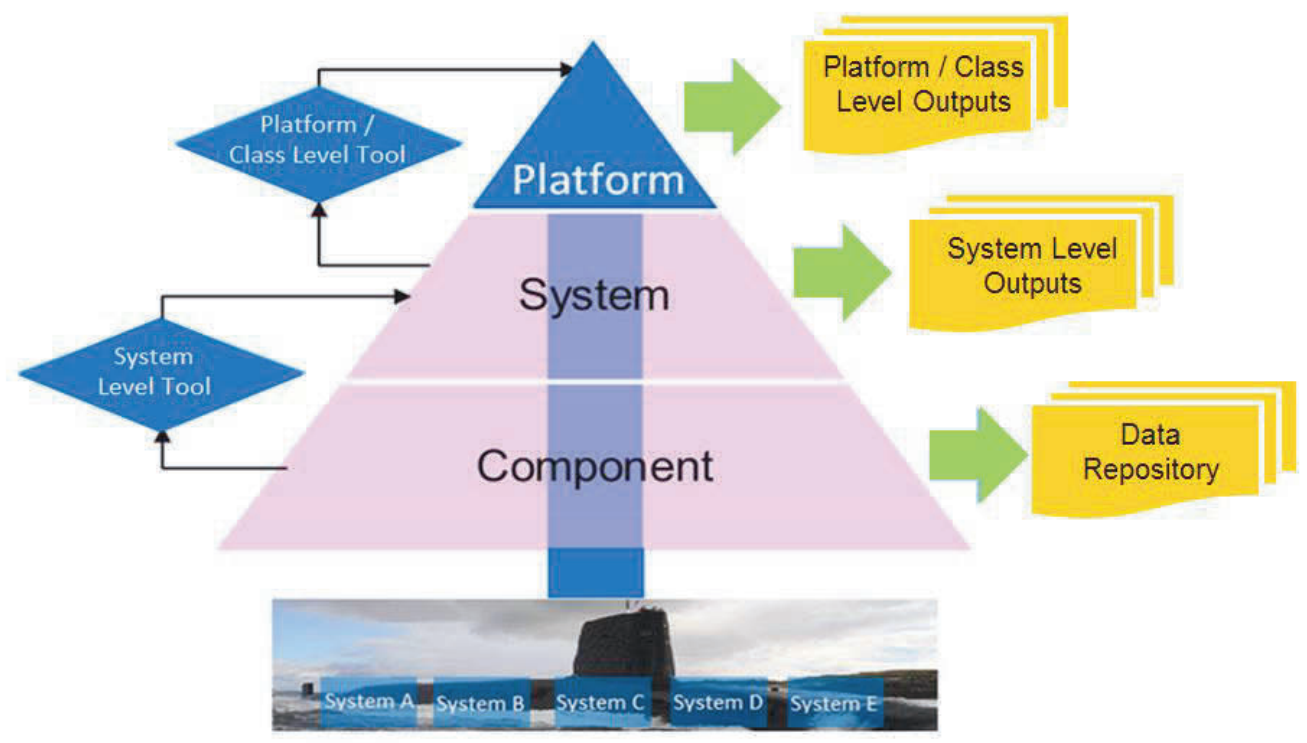

Figure 5 Modelling Data Flow

\section{Model Tailoring Approach}

A submarine and its support solution are inherently complex; being comprised of hundreds of systems and hundreds of thousands, if not millions, of components. This necessitates a tailoring approach to prioritise systems for modelling and analysis to maximise resource and cost efficiency.

\subsection{Mission and Safety Criticality}

Platform and support solution systems that perform functions critical to operational readiness and safe operation are prioritised for modelling as they have the greatest impact on the ability of a submarine class to meet the assigned through-life availability and safety targets. However, the analysis of non-critical systems is still required to inform through-life cost, and maintenance assessments. Criticality is defined as:

- A system is defined as mission critical if a loss of function would cause an immediate withdrawal from operational readiness

- A system is defined as being safety critical if its function provides a significant safety contribution to a Key Safety Functions (KSF). A KSF is a high level operation that the platform must perform to control a Major Accident Hazard (MAH), and which if lost, will result in an initiating event that could cause a MAH.

Key Safety Functions include but are not limited to:

- Vehicle Control: Control of the platform in six degrees of freedom, and ensuring watertight and structural integrity e.g. Control Steering and Diving, Control Watertight Boundary, Provide Structural Strength 
- Sustain Life: All the systems required to support the human element on the platform e.g. Control of Submarine Atmosphere

- Control Damage Hazards: Systems required to control hazards and manage damage e.g. Control Fire Hazards, Post Accident Management

- Manage Ordnance, Munitions, and Explosives: Control the MAHs associated with handling, storing, discharging and disembarking ordnance, munitions, and explosives e.g. Confine Fuels, Propellants, Oxidants and Explosives

- Power and Propel: Provides the propulsive and non-propulsive power that is required by the platform e.g. Provide Pneumatic Power, Provide Electrical Power

- Special Purpose Systems: Each platform is likely to have a specific system or capability that will have its own specific MAHs

The criticality of a system is dependent on which capabilities the platform requires in each operational state, e.g. a system may be required when at sea but not when in-standby or in a maintenance period.

\begin{tabular}{|c|c|c|c|c|c|c|}
\hline & \multicolumn{3}{|c|}{ Platform } & \multicolumn{3}{c|}{ Support Solution } \\
\hline Location & System A & System B & System C & System A & System B & System C \\
\hline Mission & Yes & Yes & Yes & & & \\
\hline Standby & Yes & Yes & & & & Yes \\
\hline Maintenance & & Yes & & Yes & & Yes \\
\hline Refit Period & & & & Yes & Yes & Yes \\
\hline
\end{tabular}

Figure 6 System Utilisation

\subsection{Component Configuration}

It is imperative to have a consistent view on what is fitted to the platform and support solution, such as a system configuration items (CI) list, to ensure analysis and modelling outputs are truly representative. This information should be held in a product configuration tool with version control. This allows for the impact of component changes to be effectively assessed against previous versions, so operational performance can be monitored through-life.

\subsection{Target Allocation}

A total operational target for the platform is assigned for availability, cost and safety, which is the contractual measure of performance. The total target is a combination of the 'Support Solution' and 'Product' targets.

- In the case of availability and safety, the target is the minimum percentage of time the functions critical to operational readiness must be able to be performed, for a specified time period. This can also be expressed in terms of a maximum corrective and preventative maintenance downtime limit

- For cost, the target is the total expenditure, of personnel and materials, used to maintain the functions critical to operational readiness, for a specified time period that must not be exceeded

The allocated targets are apportioned down to each Product Breakdown Structure (PBS) node, to allow for assessments against system specific targets. For availability and safety, only mission or safety critical systems require target allocation. For cost, all nodes must be considered. 


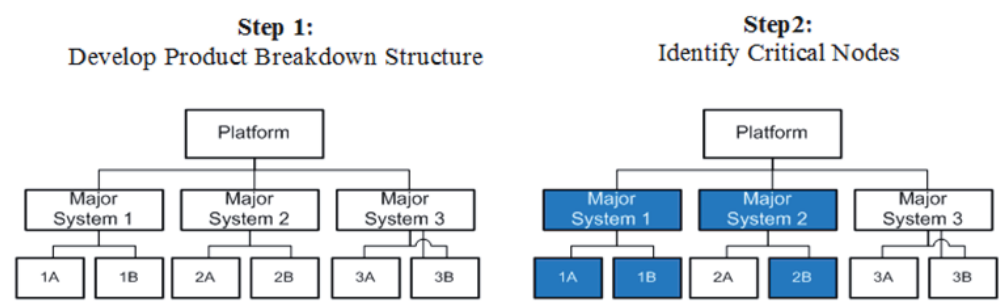

Figure 7 Target Allocation Process

Targets are allocated equally in the first instance and are adjusted as required on a case by case basis as throughout the programme. For example a major system has a requirement of $90 \%$ availability over a defined period and has three constituent systems. Percentage targets can be allocated equally to each system:

- Target $_{\text {System }}=\sqrt[\sum \text { systems }]{\text { Target }_{\text {Major System }}}$

- There are three systems; therefore the equation is $\sqrt[3]{90 \%}$ resulting in a system target of $96.55 \%$

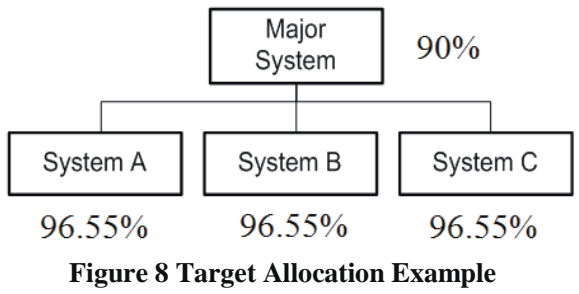

\section{Effective Modelling Prerequisites}

A set of objectives has been flown down to achieve the desired level of supportability for a class. To achieve these objectives, a major supportability analysis program can then been started, preferably as early as possible within the product lifecycle. Doing so will aid in influencing the design as necessary to make it as supportable as possible. These objectives are then transformed into processes, created and tailored by Babcock ${ }^{\circledR}$, to allow for a path to be followed when performing the necessary work.

To meet these processes, an approach has been taken to identify all the data required to meet each step. This data has been compiled into a Data Model; which is a suite of information showing what data elements are required for each process, defines what standards they are aligned to, and the format of the output data. This approach has been taken to allow for the most compliant supportability analysis possible for the MOD. Understanding the needs of what each process requires in terms of data has allowed for the tailoring of support tools to be created in order to perform the tasks required and produce the overall objectives set to Babcock ${ }^{\circledR}$. This high-level process is shown in Figure 9.

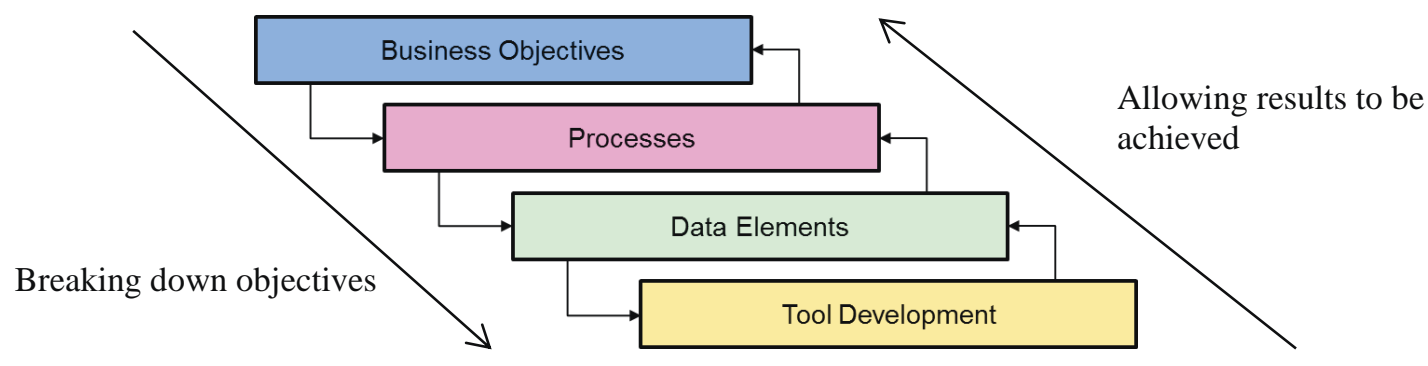

Figure 9 Modelling Tool Tailoring Process

Following this process, a vast suite of tools are being tailored and utilised to perform the supportability analysis in the most effective manner to meet the demands of the MOD. Using these tools, all of the steps within supportability analysis process shown in Figure 9 can be effectively performed. Design changes can then be recommended to the Design Authority. Being able to influence the design for greater supportability is one of the key reasons for having a Support Authority. It is far easier, and cheaper, to build support into the design of a 
product, rather than building a product and then trying to find remedies for any in-service issues, while keeping the availability of said product high. This has been a lesson from experience that the MOD is trying to remedy in future submarine programmes. The need for a highly supportable class increases the overall availability and reduces the chances of a break in the operating profile.

The intent of a Support Authority is to allow for supportability analysis to be completed across all systems within the aforementioned areas. In doing so, design changes can be proposed early in the life of designs, allowing for increases in availability, safety and a decrease in cost. The Supportability Analysis model is shown in Figure 10.

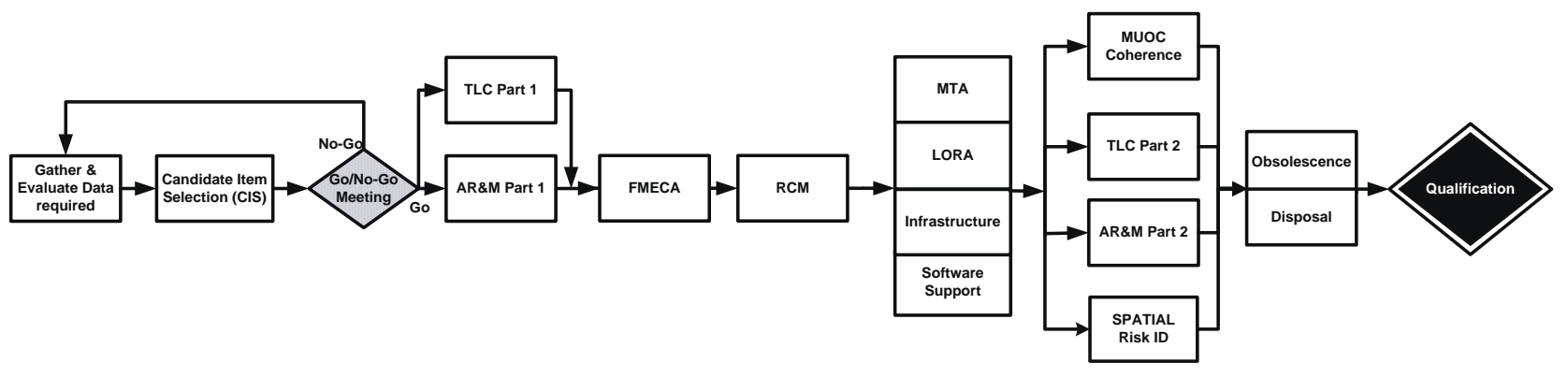

Figure 10 Supportability Analysis Process

A key area for influencing the design early is through the use of Availability, Reliability and Maintainability (AR\&M) analysis, as well as Through-life Cost (TLC) analysis. AR\&M is typically undertaken through understanding a system layout and its use, recreating this in a Reliability Block Diagram (RBD) using all the available part data (i.e. Mean Time Between Failures, Mean Time to Repair etc.) and running varying mission simulations to see the AR\&M output data. This is a key early modelling technique that allows for any potential issues in a system to be highlighted for improvement in order to meet target operational requirements. By altering the arrangement of the RBD, either by adding in higher performing parts, or adding in passive or active redundancy parts, the AR\&M characteristics can be improved through such work. TLC analysis works on a similar premise. In doing TLC analysis, suggestions can be made to summarise if systems are capable of being made and operated for a cheaper value than initially designed. Using this, and the AR\&M analysis, Trade-Off analysis can then be performed to decide what route is best to take, without impacting on each system's target performance objectives. This Trade-Off analysis forms a major basis for influencing the design. If sufficient, accurate evidence can be provided so that any proposed changes would have a greater improvement on supportability without impacting on availability, safety and cost, it shows the value added by embedding support into the design at such an early stage in the product lifecycle.

The supportability analysis process can be run throughout the entire product lifecycle in order to improve the quality of the data being performed at each stage. Having up-to-date, usable data aids in the overall performance estimations that can be taken forward into the performance modelling aspects of supportability analysis. This repeatable process allows for data maturity to increase through-life, as systems go through real-life trials and testing and move into the in-service environment. Capturing this data is one of the key processes for maturing a support solution as the product lifecycle progresses. Employing a Data Recording and Corrective Action System (DRACAS) allows for real-life data through the product lifecycle to be collated into a singular database that can then allow future supportability analysis iterations to have the most up-to-date performance data available.

Having accurate, usable data is essential for support programmes to provide the best analysis and modelling possible. Creating and utilising appropriate toolsets involved throughout a product lifecycle is an effective starting point. Implementing effective training for all involved to be trained to a standard where the data they are providing into a DRACAS system is key to realising the potential of the supportability toolsets employed. Therefore, designing a training programme where the users understand the toolsets, why they are being used, and what information is required to be populated to feed into the DRACAS system will provide a far more efficient support solution.

The collation of this ever changing data will allow for a more robust support solution to be developed through the product lifecycle. This data will allow for modelling of the support solution to be undertaken from an early stage in this lifecycle, and as data matures so too will the modelling approaches. 


\section{Modelling Methodology}

\subsection{System Level}

This section goes into detail the major tasks within a supportability analysis programme. It will guide through the process shown in Figure 10 and highlight how major system supportability information is generated from ever-evolving data to a final supportability analysis output.

\subsubsection{Source Data Confidence}

The AR\&M and cost characteristics need to be determined for every system Configuration Item (CI), at the lowest item level, in line with the required data elements from the chosen standard. Data will be sourced from a number of different locations, throughout the course of the programme, with each source implying a variable confidence on to the data it is able to generate. Each data source data type has an assigned percentage confidence band that represents the potential deviation away from the performance characteristic. The higher the source data confidence, the smaller the percentage band. This is illustrated in Figure 11 where the source data that provides the highest confidence in the performance characteristic is from the submarine at sea:

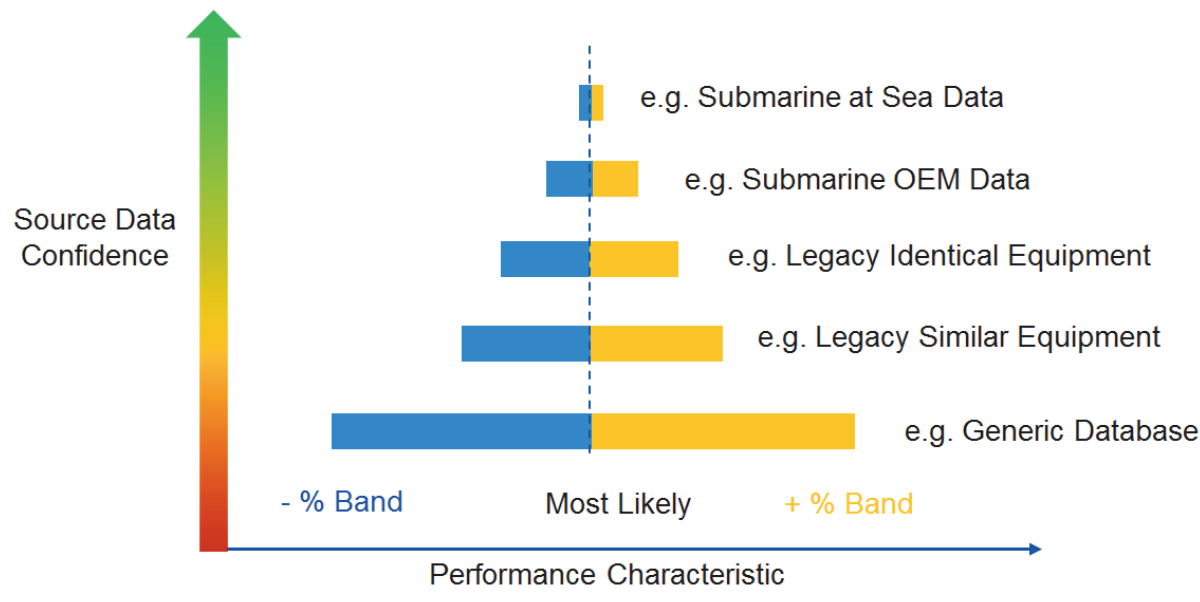

Figure 11 Source Data Confidence

\subsubsection{AR\&M and Cost - Functional/Reliability Block Diagrams (RBDs)}

A Reliability Block Diagrams (RBD) is an overall system reliability prediction model, used to forecast the ability of a system to carry out its function, by representation of the system's functional architecture, operation, and component configuration and AR\&M characteristics. Component AR\&M and cost characteristics data are sourced from the highest possible confidence data source to assure confidence in the RBD outputs. System functional architecture is deduced from system design documentation, such as system schematics and diagrams, and illustrated parts catalogues. RBDs are sub-divided to the System's constituent sections as required, such as Sub-Systems and Major Equipment. Input fields include but are not limited to:

- Component failure modes and failure rates

- Component failure distributions: the appropriate distribution must also be determined for each component to ensure the model represents each system's performance as accurately as possible e.g. 'normal', 'exponential 'or 'lognormal'.

- Preventative Maintenance (PM) tasks: Task schedule, task details, Mean Active Repair Time (MART), resource requirements - personnel skill level and infrastructure, operational phase requirement

- $\quad$ Corrective Maintenance (CM) tasks

- Cost data: spares costs, labour rates

In the case of complex system, RBDs must be able to comprehensively represent their functional characteristics to enable modelling. This could include; standby and active redundancy arrangements, using subsystem diagrams to represent a complex component or part of a system, and modelling a part of a system which is used in multiple configurations to provide the system function. An example of this is in a hydraulic system in 
which two hydraulic pumps, No.1 and No 2, can be cross connected to provide the total duty required; in this scenario there are three possible configurations which maintain system function:

- $\quad$ No. 1 and No. 2 hydraulic pumps are working

- No. 1 pump is working, No. 2 pump is down and the cross connections are open

- No. 2 pump is working, No. 1 pump is down and cross connections are open

RBDs are modelled using probabilistic modelling software (e.g. Reliasoft ${ }_{\circledast}$ BlockSim $_{\circledast}$ ). System performance, in each operational phase, is simulated over multiple runs, to account for the varying of starting condition possibilities due to each component's failure distribution. The number of runs is dependent on how long the model takes to reach the desired convergence level, time increases with model size and complexity. Figure 12 illustrates an example RBD, and key inputs and outputs:

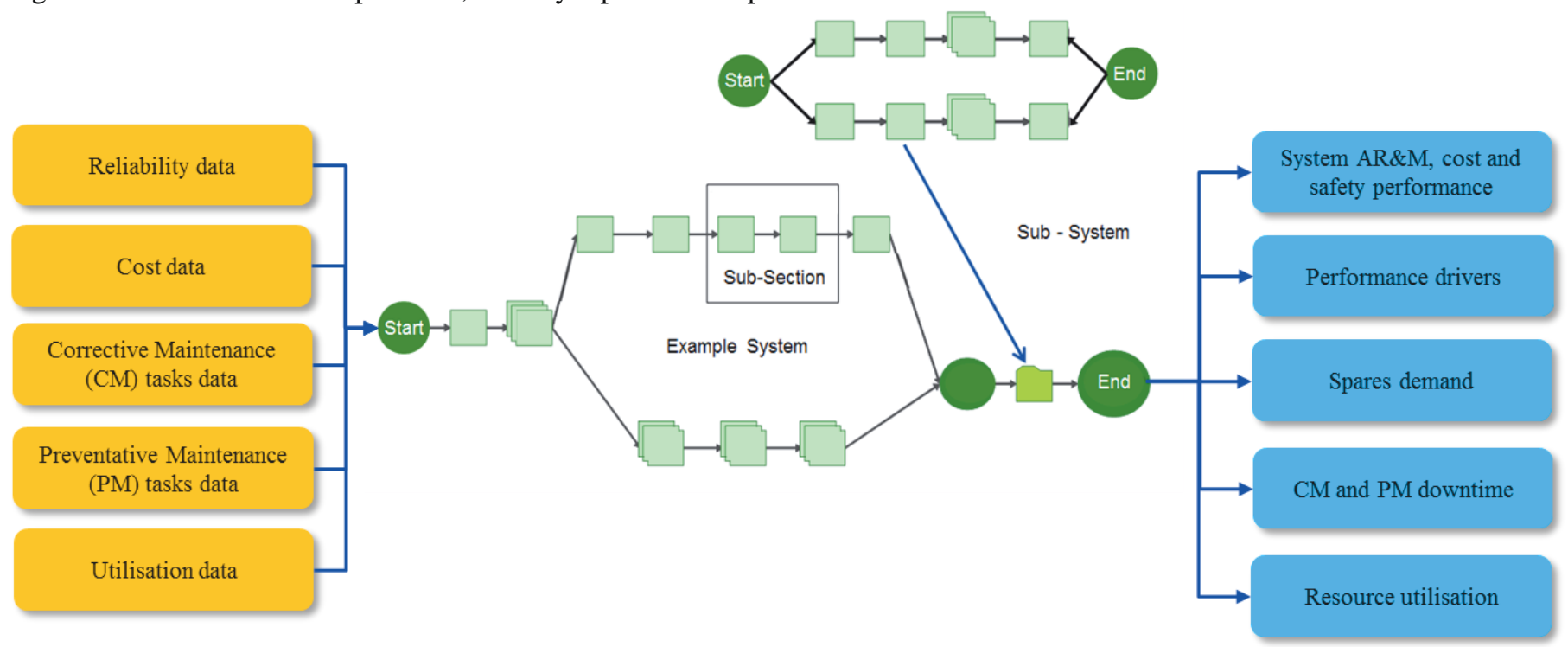

Figure 12 Functional RBD Example

Component performance metrics are used to identify the greatest impact drivers of overall system performance. Percentage bands indicate the potential deviation from the most likely predicted performance value as per source data confidence, illustrated in Figure 14.

System performance is represented by box and whisker plot. The central box represents the model variance, the intrinsic difference in outputs due to the probabilistic nature of the modelling. The size of the variance is a function of the model and the data and is comprised of $5^{\text {th }}, 50^{\text {th }}$ and $95^{\text {th }}$ percentile values from the most likely system performance input. Source data confidence is represented by the 'whiskers' which are generated from using the confidence band inputs.

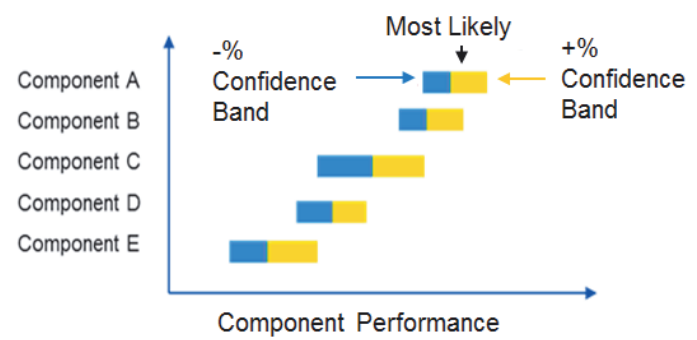

Figure 13 Component Performance Drivers

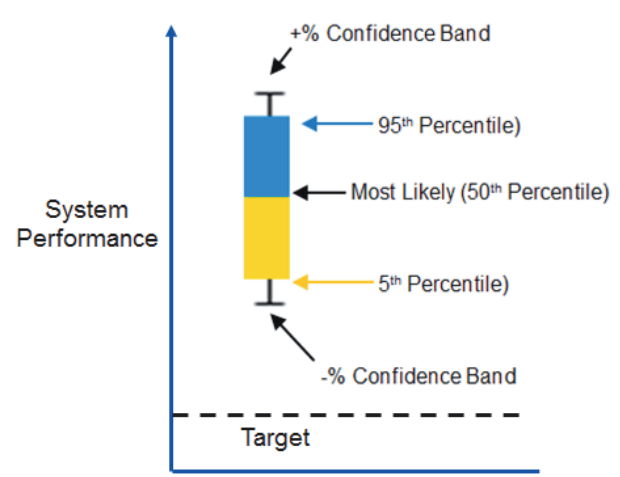

Figure 14 System Performance Boxplot

Corrective and preventative maintenance tasks can have different operational phase requirements, e.g. some activities can only be undertaken using shore facilities or in a dock, and some can be undertaken by ship staff, while others require specialist support. System functional requirements also differ in each operational phases. This necessitates creating RBDs with specific functional architecture for each phase to allow for performance outputs to be generated for all operational states. 


\subsubsection{Failure Mode, Effects, and Criticality Analysis (FMECA)}

Failure Mode, Effects, and Criticality Analysis is a method of defining the ways of why a piece of equipment might fail and the effects this will cause. It is typically performed using a risk assessment, in order to define the severity, occurrence and detection of risks. Using this methodology, a prioritization of the risks can be created and evaluated typically through the multiplication of scores against severity, occurrence, and detection to give an overall Risk Priority Number. FMECA therefore creates a basis for planning preventative maintenance, and allowing for all failure modes to be defined for any corrective maintenance required in the future. FMECA leads into RCM, as it transitions from why equipment may fail to what causes it to fail.

\subsubsection{Reliability Centred Maintenance (RCM)}

Reliability Centred Maintenance (RCM) is a process used to determine what must be done to ensure that equipment can continue to do what its users want in its present operating context. By performing RCM, it can lead to the development of unique maintenance schedules for each piece of equipment within the class. RCM identifies all of the failure modes of a piece of equipment and prioritises the importance of addressing each failure mode. The output of RCM defines a set of maintenance task candidates, and allows for the most effective solution to be chosen. RCM then provides the initial basis for MTA and breaks down what is required to maintain equipment from the method defined in RCM.

\subsubsection{Maintenance Task Analysis (MTA)}

Maintenance Task Analysis is used as means of providing how equipment will be maintained and includes the details required for all resources when performing corrective and preventative maintenance. This information is typically stored in a Logistic Support Analysis Repository (LSAR) for all equipment used. The collation of this data is typically built up through the use of Suitably Qualified and Experience Personnel (SQEP) and manufacturer's guidance, in order to define what is specifically required to perform corrective and preventative maintenance. MTA leads to information on maintenance task durations, including work-in-way and work-in-wake. It also provides information on all the tools required for each task, as well as any skills that the maintainers may require to complete said task. Having defined how equipment will be maintained, LoRA is then used to define where and how it will be maintained.

\subsubsection{Level of Repair Analysis (LoRA) / economic LoRA (eLoRA)}

Level of Repair Analysis follows on from MTA and determines the best option for where and how an item will be repaired, replaced, or discarded. Although options can sometimes be too expensive to undertake for the size of the task, this is where Economic Level of Repair Analysis (eLoRA) is performed. eLoRA takes into account economic factors surrounding each maintenance task. Through a series of trade-off analysis that is formed from the AR\&M and TLC analysis, the best cost-effective solution for maintaining each piece of equipment can then be derived.

\subsection{Platform / Class Level}

This section outlines how the culmination of system level analysis for the design and support solution can be utilised to model availability, cost, and safety performance at platform and class level, to provide operational capability assurance. Topics include:

- Maintenance Planning: The assessment and optimisation of all maintenance tasks within each operational phase, to ensure they can be undertaken within resource constraints to maintain the required readiness level - mission, standby, maintenance period etc.

- Spatial Modelling: Utilising a whole submarine spatial model to assess maintenance envelopes and removal routes to ensure maintenance can be undertaken within the required timeframe

- $\quad$ Platform / class Availability, Safety and Cost Prediction Model 


\subsubsection{Maintenance Planning}

Maintenance planning is undertaken to distinguish whether major maintenance tasks can be aligned to submarine MUOC patterns. Being able to do so can allow for the correct resources - such as manning and spares - to be in place in advance of a submarine coming for a certain maintenance period. Due to various types of systems used on board, there has to be a given level of priority for some systems at different stages in the lifecycle. Therefore, pre-planning the maintenance can alleviate any lack of resource and planning for maintenance tasks. This defines a working schedule for maintenance periods, allowing for operating profiles to be met, and keeping availability high and costs down.

\subsubsection{Spatial Model}

Spatial Analysis is one of the major Supportability Analysis tasks on a naval platform. Using the 3D model from the designer, a team of Spatial Analysts can then go through the model and highlight any areas where users may be blocked when accessing equipment. It can also be used to show of any clashes in equipment within the model that would cause an issue in build. Spatial Analysis can quickly highlight issues such as the need for rerouting some pipework in order to access a pump. If the pipework would require being isolated and removed to get to the pump, and there is a viable option of rerouting the pipework to gain better access, then Spatial Analysis will highlight this. If it is possible to make a number of small design changes, to reduce the time to access equipment and allow operators and maintainers to quicker access, then it makes a far greater improvement on availability through-life. Having SQEP, in particular those who have worked on a similar naval platform can allow for a number of spatial issues to be highlighted which can influence any required design changes.

The use of 3D Computer Aided Design (CAD) models has vastly improved the efficiency at which spatial issues are resolved. However, with advances in Virtual Reality (VR) technology, there is now the option of having operators, maintainers, and SQEP "walk" throughout the platform and highlight any concerns. This is proving to be a far more effective method for performing Spatial Analysis, and is far more successful at highlighting any access issues on board. The ability to spot clashes prior to manufacture can save major rework in the future, where the possibility of concessions can reduce the performance, while increasing the costs associated. Figure 15 highlights two example removal route that would need to be assessed to determine if each corresponding maintenance task can be undertaken within the required time constraints:

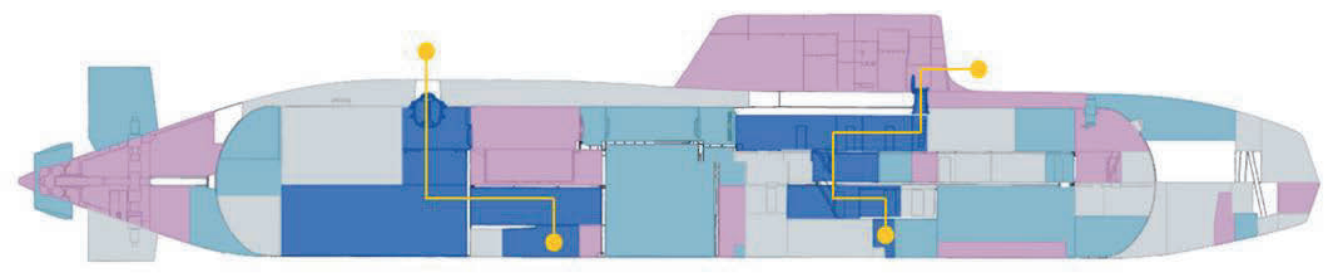

Figure 15 Astute Spatial Model Example

\subsubsection{Class Level: Availability, Safety and Cost Prediction Model}

Class level modelling is used to forecast the ability of the submarine class, and support solution, to deliver the target operational capability over a defined duration, and identify and prioritise risks, to optimise performance. The performance characteristics of all mission and safety critical systems/functions AR\&M and cost performance characteristics are used as model inputs e.g. the derived System MTBFs and MARTs from RBDs modelled using probabilistic modelling software.

The model in use by Babcock ${ }_{\circledast}$ is called the "Support Upkeep Performance Prediction Operational Reliability Tool - Simulation". SUPPORT Sim is based on a commercial package which is heavily customised to model the probabilistic performance of a class of submarines over their service lives. The tool is capable of taking the AR\&M characteristics of the constituent systems of a platform, derived from the system level analysis (e.g. RBDs), applying the restrictions associated with working within a support solution, and using an event-based simulation to predict performance over a defined Maintenance, Upkeep, and Operational Cycle (MUOC). It uses a "Monte Carlo" approach to generate event timings for each simulation run based on the input failure mode distributions. Multiple simulation runs, known as histories, are amalgamated to give improved accuracy in overall results.

A MUOC provides a location and readiness level schedule of each platform for a specified time period; a 5 platform example is shown in Figure 16. It identifies the task each platform is undertaking at a given time, and in-turn specifies what decisions can be made when a failure event occurs to maintain mission capability, in line with operational doctrine. Interactions between each platform, such as responses to platform failure situations, 
are defined and replicated to more closely predict operational performance. E.g. a standby platform raised mission readiness early due to a failure on the deployed platform, or platform mission duration extended due to a failure in standby.

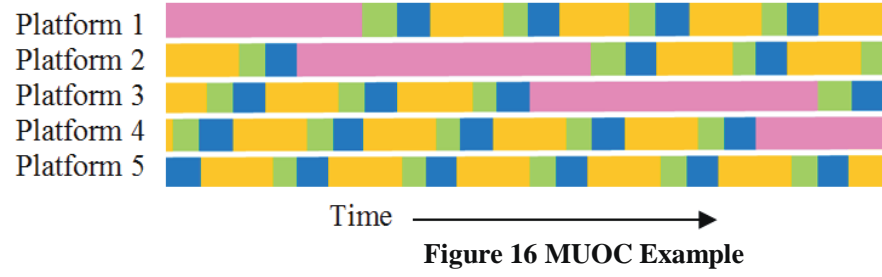

Mission

Standby

Maintenance

Refit Period

Figure 16 MUOC Example

Key outputs of the model, as shown in Figure 17, include but are not limited to a prediction of class level availability, cost, and safety performance, and the identifying driving systems and functions from the design and support solution that impact class level performance. Coherence to each MUOC task is also assessed to provide programme assurance, and highlight areas where there is a risk of task deviation such maintenance tasks overruns or potential failure modes.

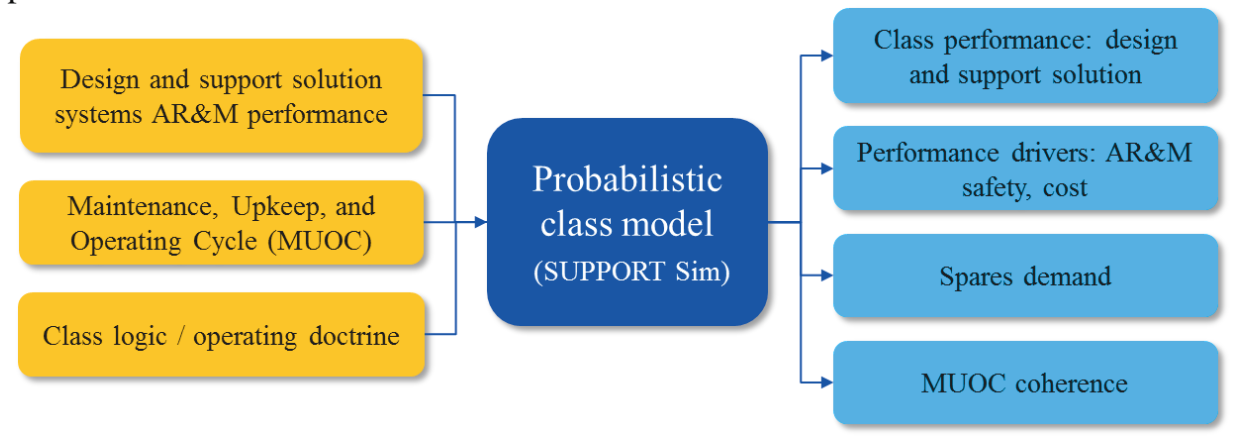

Figure 17 Probabilistic Class Modelling

Platform and class modelling can be utilised at every stage of a Submarine's life cycle to provide assurance that requirement targets will be met. As the design phase progresses, outputs are used to optimise decisions made in the support solution design against constraints in the platform design. Decisions could include but are not limited to preventative maintenance prioritisation, component spares levels, number of personnel, and system design changes to improve functional performance. The effect of changes can be modelled pre implementation to inform the business case, and post implementation to validate that the change had the desired effect, ensuring capability is maximised whilst working within greater financial constraints.

As the platform and support solution design progresses through-life, higher confidence data sources become available: e.g. Original Equipment Manufacturer (OEM) AR\&M data as the design matures, and DRACAS feedback data from testing of new components. These new data sources are incorporated into the model as they become available, increasing confidence in the model outputs, illustrated in Figure 18. A greater data confidence allows for more effectively decisions to be made to optimise the design and support solution, to maximise capability and minimise cost. 


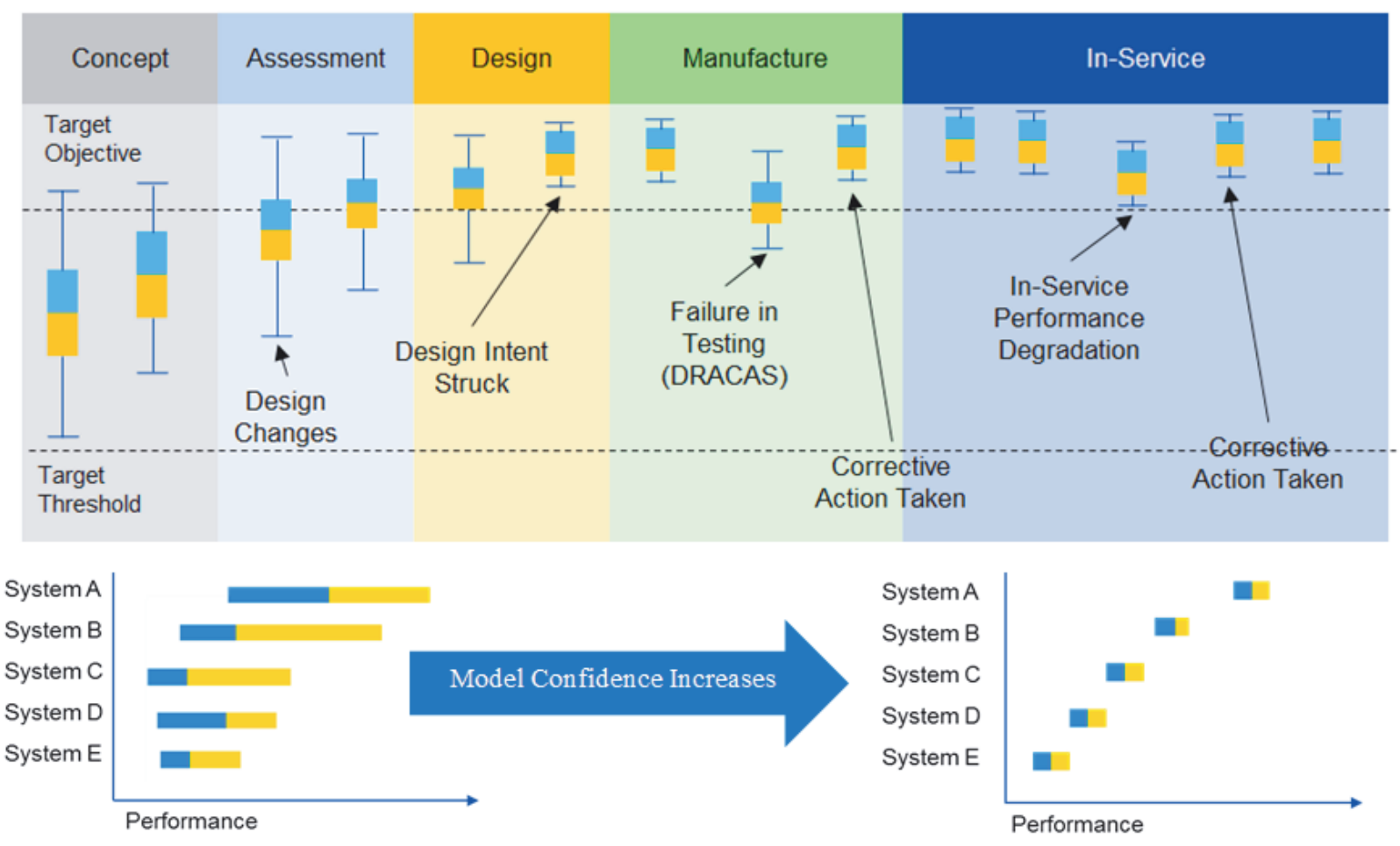

Figure 18 Class Modelling Through-life 


\section{Conclusion}

Support is too often unjustly negated when designing a product prior to it going into service. Building support into the design early proves to be far cheaper the earlier it is implemented. By designing and modelling a support solution early on in a product lifecycle, there is increased opportunity to influence the design of a submarine to allow UK MOD to have a submarine which is available when required, have it safe to operate by the crew and maintenance staff, and to be affordable through-life. This is more than sending submarines out on a mission, and requires an effective support solution to be in place to allow the class of submarines to be able to complete their missions, in accordance with the MUOC cycle.

In order to achieve this, the support Technical Authority has to be given the opportunity to positively influence the design to meet the three main objectives above. Being able to provide a platform with support built into the design can pave a way for all future platforms being designed for the UK MOD. In the current economic climate, where budgets for the Navy are decreasing, there is a real opportunity to show that a capable submarine can be designed, built, and deployed on service while not breaking the bank by incorporating as much supportability elements into the design as possible in order to decrease costs, while still meeting all of the require capabilities that the UK MOD requires.

High quality, up-to-date data needs to be used throughout the product lifecycle to allow the support solution modelling to be as accurate and effective as possible going forward. Training solutions have to be in place and renewed and updated through time to allow all involved the ability to provide the best data possible, in order to keep the submarines available, safe, and cost effective though life. Creating a support solution capable of capturing this data and optimising the training involved is the next major step within the current programme. Having a DRACAS solution as the submarines move into manufacture can allow for corrective actions to be implemented allowing target objectives to be met as quickly as possible. This allows the supportability analysis to be updated to reflect the ever-evolving platform state as to improve the data maturity for the support solution. The support solution aspects will be expanded on in a future iteration of the paper.

It is recommended to build support into the design as early as possible to allow submarines to complete the missions required of them through life. Being involved early saves on costs, and increases the availability and safety of a platform. Increasing the awareness of how vital support is will allow future generations of submarines to be optimally designed to achieve the greatest levels of availability, safety and cost through the realisation that support is key to allowing each submarine to fulfil their missions. 


\section{References}

HM Government, 2015. National Security Strategy and Strategic Defence and Security Review 2015. [Online] Available at:

https://assets.publishing.service.gov.uk/government/uploads/system/uploads/attachment_data/file/555607/2015 Strategic_Defence_and_Security_Review.pdf

Kongsberg, 2017. K-Sim Support - Product life-cycle\& portingplans. [Online]

Available at: https://www.kongsberg.com/ /media/Kongsberg\%20Digital/Simulation/UC2017/K-

Sim\%20Support_Lars\%20Klemmetsby_Kongsberg\%20Digital.ashx?la=en

Mani, N., 2011. Impact of Design Cost on Design Bid Build Project Performance. [Online]

Available at: https://digitalscholarship.unlv.edu/cgi/viewcontent.cgi?article=1999\&context=thesesdissertations

\section{Glossary of Terms}

\begin{tabular}{|c|c|}
\hline Abbreviation & Definition \\
\hline AR\&M & Availability, Reliability, and Maintainability \\
\hline CAD & Computer Aided Design \\
\hline $\mathrm{CI}$ & Configuration Item \\
\hline $\mathrm{CM}$ & Corrective Maintenance \\
\hline DRACAS & Data Recording and Corrective Action System \\
\hline eLoRA & Economic Level of Repair Analysis \\
\hline FMECA & Failure Mode Effect and Criticality Analysis \\
\hline GDEB & General Dynamics Electric Boat \\
\hline KSF & Key Safety Function \\
\hline LoRA & Level of Repair Analysis \\
\hline LSAR & Logistic Support Analysis Repository \\
\hline MAH & Major Accident Hazard \\
\hline MOD & Ministry of Defence \\
\hline MTA & Maintenance Task Analysis \\
\hline MTBF & Mean Time Between Failures \\
\hline MART & Mean Active Repair Time \\
\hline MUOC & Maintenance, Upkeep, and Operational Cycle \\
\hline ODC & Oman Drydock Company \\
\hline OEM & Original Equipment Manufacturer \\
\hline PBS & Product Breakdown Structure \\
\hline PM & Corrective Maintenance \\
\hline RBD & Reliability Block Diagram \\
\hline $\mathrm{RCM}$ & Reliability Centred Maintenance \\
\hline SDSR & Strategic Defence Security Review \\
\hline SQEP & Suitably Qualified and Experience Personnel \\
\hline TLC & Through Life Cost \\
\hline VR & Virtual Reality \\
\hline
\end{tabular}

\section{Liability}

The opinions expressed in this paper are those of the authors and not necessarily those of Babcock International $_{\circledast}$ or the UK MOD. 